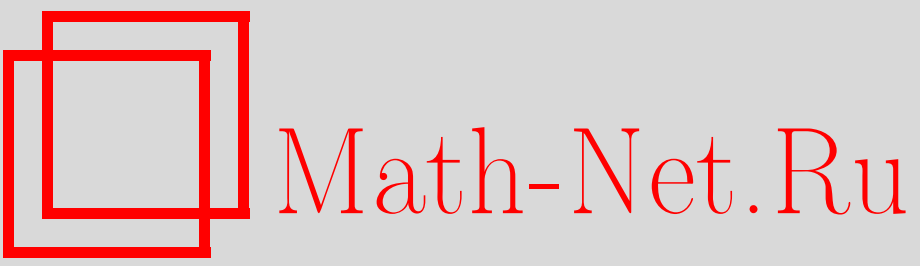

Б. С. Кругликов, Препятствия к построению псевдоголоморфных отображений, УМН, 1997, том 52, выпуск 3, 167-168

DOI: https://doi.org/10.4213/rm854

Использование Общероссийского математического портала Math-Net.Ru подразумевает, что вы прочитали и согласны с пользовательским соглашением

http://www.mathnet.ru/rus/agreement

Параметры загрузки:

IP: 54.174 .149 .18

26 апреля 2023 г., 15:22:12 


\title{
ПРЕПЯТСТВИЯ К ПОСТРОЕНИЮ ПСЕВДОГОЛОМОРФНЫХ ОТОБРАЖЕНИЙ
}

\author{
Б. С. Кругликов
}

Пусть $\left(L^{2 l}, j_{L}\right)$ и $\left(M^{2 m}, j_{M}\right)$ - почти комплексные многообразия, т.е. многообразия, снабженные автоморфизмами $j_{L}$ и $j_{M}$ касателшных пространств, удовлетворяющих $j_{L}^{2}=$ $-\mathbf{1}_{L}: T_{*} L \rightarrow T_{*} L$, и $j_{M}^{2}=-\mathbf{1}_{M}$. Псевдоголоморфиным отображением $u: L \rightarrow M$ назьвается такое отображение, что его дифференциал $\left.u_{*}\right|_{x}=d u_{x}: T_{x} L \rightarrow T_{u(x)} M$ коммутирует с почти комплексной структурой: $j_{M} \circ u_{*}=u_{*} \circ j_{L}$.

Обозначим через $\mu=\mu_{x}$ идеал в кольце гладких функций $C^{\infty}(L)$, обращающихся в точке $x$ в нуль. Степень этого идеала $\mu^{k}$ является идеалом в кольц функций, обращающихся в нуль в точке $x$ вместе со своими производньми порядка $<k: \mu^{k}=\left\{f\left|\partial^{\sigma} f(x)=0,\right| \sigma \mid<k\right\}$. Будем обозначать через $\mu^{k}=\mu^{k} T(p, q)$ подмодуль в модуле тензоров типа $(p, q)$, имеющих в точке $x$ нуль порядка $k$. Обозначим символом $\mathscr{O}_{L}(x)$ росток окрестностей точки $x \in L$. Обозначим также $\mu=\mu_{x, y}=\left\{\Phi: T \mathscr{O}_{L}(x) \rightarrow T \mathscr{O}_{M}(y) \mid \operatorname{Im} \Phi \subset \mu_{y}\right\}$ и, соответственно, $\mu^{k}=\mu_{x, y}^{k}$. Формальным псевдоголоморфным отображением назьвается отображение $u: \mathscr{O}_{L}(x) \rightarrow \mathscr{O}_{M}(y)$ такое, что $j_{M} \circ u_{*}-u_{*} \circ j_{L} \in \mu_{x, y}^{\infty}$. Введем пространство струй $J_{P H}^{k}$ как подмножество в $J^{k}(L, M)$ :

$$
J^{k}(L, M)_{x, y} \supset\left(J_{P H}^{k}\right)_{x, y}=\frac{\left\{u_{*}: T \mathscr{O}_{L}(x) \rightarrow T \mathscr{O}_{M}(y) \mid j_{L} \circ u_{*}-u_{*} \circ j_{M} \in \mu_{x, y}^{k}\right\}}{\left\{u_{*}: T \mathscr{O}_{L} \rightarrow T \mathscr{O}_{M} \mid u_{*} \in \mu_{x, y}^{k}\right\}} .
$$

В отличие от $J_{P H}^{1}$ множество $J_{P H}^{k}, k \geqslant 2$, вообще говоря, не имеет структуры многообразия. Это связано с тем, что для отображения $\pi_{P H}^{r, s}$ ограничения проекции $\pi^{r, s}: J^{r}(L, M) \rightarrow$ $J^{s}(L, M), r \geqslant s$, прообраз точки может быть пуст. Обозначим через $\mathscr{E}^{k}$ образ проекции $\operatorname{Im} \pi_{P H}^{k, 1} \subset J_{P H}^{1} \subset J^{1}(L, M)$. Уравнение $\mathscr{E}^{1}=J_{P H}^{1}$ расслоено над $J^{0}=L \times M$ со слоем

$$
\mathscr{E}_{x, y}^{1}=\left(\pi_{P H}^{1,0}\right)^{-1}(x, y)=\left\{\Phi: T_{x} L \rightarrow T_{y} M \mid j_{M} \circ \Phi=\Phi \circ j_{L}\right\} .
$$

Рассмотрим тензоры Ниенхейса $N_{j_{L}}$ и $N_{j_{M}}$ на многообразиях $L$ и $M$ :

$$
N_{j}(\xi, \eta)=[j \xi, j \eta]-j[j \xi, \eta]-j[\xi, j \eta]-[\xi, \eta] .
$$

Непосредственно из определения следует, что если $u: L \rightarrow M$-псевдоголоморфное отображение, то $N_{j_{M}} \circ u_{*}^{\wedge 2}=u_{*} \circ N_{j_{L}}$. Более того, это равенство выполнено в точке $x$, если $u$ сохраняет почти комплексную структуру с точностью до второго порядка: $j_{M} \circ u_{*}-u_{*} \circ j_{L} \in \mu_{x, y}^{2}$.

Теорема 1. Ограничение проекиии $\pi_{P H}^{1,0}$ на уравнение $\mathscr{E}^{2} \subset J_{P H}^{1}$ имеет прообразы

$$
\mathscr{E}_{x, y}^{2}=\left\{\Phi: T_{x} L \rightarrow T_{y} M \mid j_{M} \circ \Phi=\Phi \circ j_{L}, N_{j_{M}} \circ \Phi^{\wedge 2}=\Phi \circ N_{j_{L}}\right\}
$$

Теорема 2. Пусть отображсение $u: \mathscr{O}_{L}(x) \rightarrow \mathscr{O}_{M}(y)$ таково, что

$$
j_{M} \circ u_{*}=u_{*} \circ j_{L}\left(\bmod \mu_{x, y}^{k}\right), \quad N_{j_{M}} \circ u_{*}^{\wedge 2} \equiv u_{*} \circ N_{j_{L}}\left(\bmod \mu_{x, y}^{k}\right), \quad 1 \leqslant k \leqslant \infty .
$$

В этом (и только в этом) случае существует отображение $\widetilde{u}: \mathscr{O}_{L}(x) \rightarrow \mathscr{O}_{M}(y)$ такое, что

$$
j_{M} \circ \widetilde{u}_{*}-\widetilde{u}_{*} \circ j_{L} \in \mu_{x, y}^{k+1}, \quad u_{*}-\widetilde{u}_{*} \in \mu_{x, y}^{k} .
$$

В частности, если $\left(u_{*}\right)_{x} \neq 0$, то $\left(\widetilde{u}_{*}\right)_{x} \neq 0$, а если $\left(u_{*}\right)_{x}$ - вложение или отображсение "на", то жее верно и для $\left(\widetilde{u}_{*}\right)_{x}$.

Работа вьполнена при частичной поддержке Российского фонда фундаментальных исследований (грант № 96-010710). 
СледствиЕ (Формальная часть теоремы Ньюлендера-Ниренберга, см. [3], [4]). Пусть тензоры Ниенхейса являются малыми порядка $k$ в точках $x \in L u y \in M: N_{j_{L}} \in \mu_{x}^{k}$, $N_{j_{M}} \in \mu_{y}^{k}, 1 \leqslant k \leqslant \infty$. Тогда существует отображение $u: \mathscr{O}_{L}(x) \rightarrow \mathscr{O}_{M}(y)$ такое чmo $d u_{x} \neq 0$ u

$$
j_{M} \circ u_{*}-u_{*} \circ j_{L} \in \mu_{x, y}^{k+1} .
$$

Теорема 3. Рассмотрим алгебру инвариантов $\mathscr{A}_{j}$ почти комплексной структуры $j$, определяющей "суженное" уравнение Коши-Римана $\mathscr{E}{ }^{\infty}=\left\{\Phi: T_{*} L \rightarrow T_{*} M \mid\right.$ $\left.\mathscr{A}_{j_{M}} \circ \Phi^{\wedge \star}=\Phi \circ \mathscr{A}_{j_{L}}\right\}$. Рассмотрим алгебру Ниенхейса $\mathscr{A}_{j}^{\mathcal{N}}$ - замыкание относительно скобки Ниенхейса и коммутатора (см. [1], [2]) алгебры инвариантов $\mathscr{A}^{1}=\langle j\rangle$, где j рассматривается как векторнозначная 1-форма. Эта алгебра не совпадает со всей алгеброй инвариантов, $\mathscr{A}_{j}^{\mathcal{N}} \neq \mathscr{A}_{j}$, т.е. существуют новые инварианты - висшие тензоры Ниенхейса - такие, что $\mathscr{A}_{j}=\left\langle j, N_{j}, N_{j}^{(2)}, \ldots\right\rangle$.

Теорема 4. Для любого тензора $N \in \wedge^{2} T_{x}^{*} L \otimes T_{x} L$ на фиксированном касательном пространстве $T_{x} L$ такого, что $N(\xi, \eta)=-N(\eta, \xi)=j N(j \xi, \eta)$, существует почти комплексная структура $j$ в $\mathscr{O}_{L}(x)$ такая, что $N=\left(N_{j}\right)_{x}$.

Связность $\nabla$ на $L$ определяет отображение $d_{\nabla}^{p}: \Omega^{q} \otimes \mathscr{D} \rightarrow \Omega^{q} \otimes S^{p} \Omega^{1} \otimes \mathscr{D}$, где $\mathscr{D}$ - модуль векторных полей, а $\Omega^{q}$ - модуль внешних форм на $L$.

ОПРеДЕЛЕниЕ 1. Скажем, что почти комплексная структура $j$ на $\mathscr{O}_{L}$ имеет общий вид в точке $x$, если для соответствующего тензора Ниенхейса справедливо следующее свойство отделимости: для почти любого вектора $\xi \in T_{x} L$ из $N_{j}(\xi, \eta)=0, \eta \in T_{x} L$, следует $\eta \in \mathbb{C} \xi=\langle\xi, j \xi\rangle$. Более того, будем считать, что пара $\left(\mathscr{O}_{L}(x), j\right)$ не изоморфна $\left(\mathscr{O}_{L}(x),-j\right)$.

Теорема 5. Предположим, что тензоры Ниенхейса почти комплексных структур $j_{L}$ на $\mathscr{O}_{L}(x)$ и $j_{M}$ на $\mathscr{O}_{M}(y)$ имеют общий вид. Предположим, что существует отображсние $u: \mathscr{O}_{L}(x) \rightarrow \mathscr{O}_{M}(y)$, сопрягающее $N_{j_{L}}$ c $N_{j_{M}}$ и являющееся диффеоморфизмом на образ. Тогда и является либо псевдоголоморфньм, либо антипсевдоголоморфным отображением: $j_{M} \circ u_{*}= \pm u_{*} \circ j_{L}$. Если тензоры Ниенхейса сопряжены формально, $N_{j_{M}} \circ u_{*}^{\wedge 2} \equiv u_{*} \circ N_{j_{L}}\left(\bmod \mu_{x, y}^{\infty}\right)$, и размерности многообразий $L$ и $M$ совпадают, $2 l=2 m$, то почти комплексная структура $j_{L}$ формально әквивалентна ровно одной из структур $j_{M}$ или $\left(-j_{M}\right)$.

Рассмотрим почти комплексную структуру $j$ и ее тензор Ниенхейса $N_{j}$. Он определяет билинейное кососимметричное отображение $N_{j}: \mathscr{D} \otimes \mathscr{D} \rightarrow \mathscr{D}$ на модуле $\mathscr{D}$ векторных полей.

ОПрЕДЕЛЕНИЕ 2 . Назовем почти комплексную структуру $j$ на $L$ лиевой, если ее тензор Ниенхейса $N_{j}$ определяет структуру алгебры Ли на модуле $\mathscr{D}$.

Теорема 6. Лиева почти комплексная структура $j$ задает разрешимую алгебру Ли $\mathscr{G}=\left(\mathscr{D}, N_{j}(\cdot, \cdot)\right)$ с рангом разрешимости 2: $\mathscr{G}^{(2)}=0$. Лиевь почти комплексные структурь общего вида эквивалентны или антиәквивалентны (формально или гладко), если эквивалентны соответствующие алгебры Ли $\mathscr{G}$ и связывающее сечение $\sigma \in \operatorname{Hom}\left(\mathscr{G} / \mathscr{G}^{(1)}, \mathscr{G}^{(1)}\right)$ равно нулю.

Автор благодарит профессора В. В. Льчагина за внимание к работе и полезные обсуждения.

\section{СПИСОК ЛИТЕРАТУРЫ}

[1] Алексеевский Д. В., Виноградов А. М., Льчагин В. В. // Итоги науки и техники. Современные проблемы математики. Фундаментальные направления. Геометрия 1. Т. 28. М.: ВИНИТИ, 1988. [2] Frölicher A., Nijenhuis A. // Proc. Koninkl. Nederl. Akad. Wetensch. Ser. А. 1956. V. 59. № 3. Р. 338-359. [3] Ньюлендер А., Ниренберг Л. // Математика. 1959. T. 3. №6. C. 131-144. [4] Nijenhuis A., Wolf W. // Ann. Math. 1963. V. 77. P. 424-489.

Московский государственньй университет им. М.В. Ломоносова E-mail: borkru@difgeo.math.msu.su
Принято редколлегией 29.10.1996 